\title{
Treatment of post-craniotomy acute severe supraorbital neuralgia using ultrasound-guided pulsed radiofrequency: a case report
}

This article was published in the following Dove Press journal: Journal of Pain Research

\author{
Xiong Xiao ${ }^{1, *}$ \\ Hao Ren ${ }^{2, *}$ \\ Nan Ji' \\ Fang Luo ${ }^{2}$ \\ 'Department of Neurosurgery, Beijing \\ Tiantan Hospital, Capital Medical \\ University, Beijing 100050, People's \\ Republic of China; 'Department of \\ Anesthesiology and Pain Management, \\ Beijing Tiantan Hospital, Capital \\ Medical University, Beijing 100050 , \\ People's Republic of China \\ *These authors contributed equally to \\ this work
}

Correspondence: Fang Luo Department of Anesthesiology and Pain Management, Beijing Tiantan Hospital, Capital Medical University, Beijing 100050, People's Republic of China Tel +861067096664 Fax +86 I06 7050177 Email luofangwt@yahoo.com

\begin{abstract}
Pulsed radiofrequency is an effective, safe, and nondestructive pain treatment technique that has been insufficiently reported in the treatment of supraorbital neuralgia. We report the successful use of pulsed radiofrequency to alleviate acute severe supraorbital neuralgia in a post-craniotomy patient. A 52-year-old female, who had acute severe supraorbital neuralgia after having received a right frontal craniotomy and with poor efficacy in response to medication and a nerve block, was subjected to ultrasound-guided pulsed radiofrequency treatment and experienced complete pain relief, as a result of which the patient stopped taking all analgesic drugs. The patient had follow-up visits for 1 year, during which she reported no pain. Furthermore, no side effects of nerve damage after pulsed radiofrequency were observed. Our results suggest that pulsed radiofrequency is a promising technique for the treatment of supraorbital neuralgia and that further studies of this technique should be conducted.
\end{abstract}

Keywords: supraorbital neuralgia, pulsed radiofrequency, post-craniotomy

\section{Introduction}

Supraorbital neuralgia has a low prevalence and is infrequently covered in the literature; therefore, it is not well understood by many clinicians. ${ }^{1}$ Many patients do not exhibit clear recoveries with common medications. ${ }^{2}$ Among several therapeutic approaches, nerve block treatments do not confer lasting efficacy. ${ }^{2,3}$ Neurolysis can cause significant trauma that likely aggravates facial symptoms. ${ }^{4}$ Nerve ablation irreversibly damages nerves and can correspondingly result in decreased quality of live. ${ }^{3}$ Peripheral nerve stimulation has a considerable likelihood of complications. ${ }^{4}$ As such, current treatments cannot simultaneously achieve both satisfactory outcomes and injury reduction.

Pulsed radiofrequency is an effective, safe, and nondestructive pain treatment technique that is minimally invasive, has few side effects, and involves simple procedures. $^{5-7}$ Two published articles reported that patients with supraorbital neuralgia exhibited pain relief after at least two treatments with pulsed radiofrequency. ${ }^{8,9} \mathrm{We}$ report that a post-craniotomy patient who suffered acute severe supraorbital neuralgia got subsequent alleviation of her pain after just one treatment of pulsed radiofrequency. These results provide a reference for the future treatment of supraorbital neuralgia.

\section{Ethics approval and consent to participate}

Written informed consent was obtained for the publication of this case report and the accompanying images from the patient who participated in this study. 


\section{Case}

The patient was a 52-year-old female and was hospitalized because of "repeated dizziness for over 2 months". She was diagnosed with meningioma in the right anterior cranial fossa (Figure 1A and $\mathrm{B}$ ) and received a craniotomy and a tumor resection through an intra-hairline coronal incision (Figure 1C and D). The patient experienced satisfactory recovery in the early postoperative phase but had pain in the right eyebrow and forehead on postoperative day 3. A physical examination identified right supraorbital notch tenderness. Head computed tomography and magnetic resonance imaging generated images consistent with post-craniotomy conditions. The patient was subjected to an oral regimen of ibuprofen, oxycodone, and carbamazepine for 7 days. The patient initially experienced remission, but her headache gradually aggravated and later manifested a visual analog scale (VAS, 0 indicating no pain at all, 10 indicating the most severe pain imaginable) of 9. On postoperative day 12, the patient received steroids and local anesthetics to facilitate the treatment of a supraorbital nerve block, which resulted in complete remission of the headache right after the blockade (VAS $=0$ ). However, when the anesthetic effect disappeared, the intensity of the headache was only slightly alleviated for 2 days, which was corroborated by a VAS score of 6 , after which the VAS reached its pretreatment level of 9 . We explained the procedure, potential efficacy, and side effects of pulsed radiofrequency treatment before performing pulsed radiofrequency of the right supraorbital nerve on this patient with informed consent.
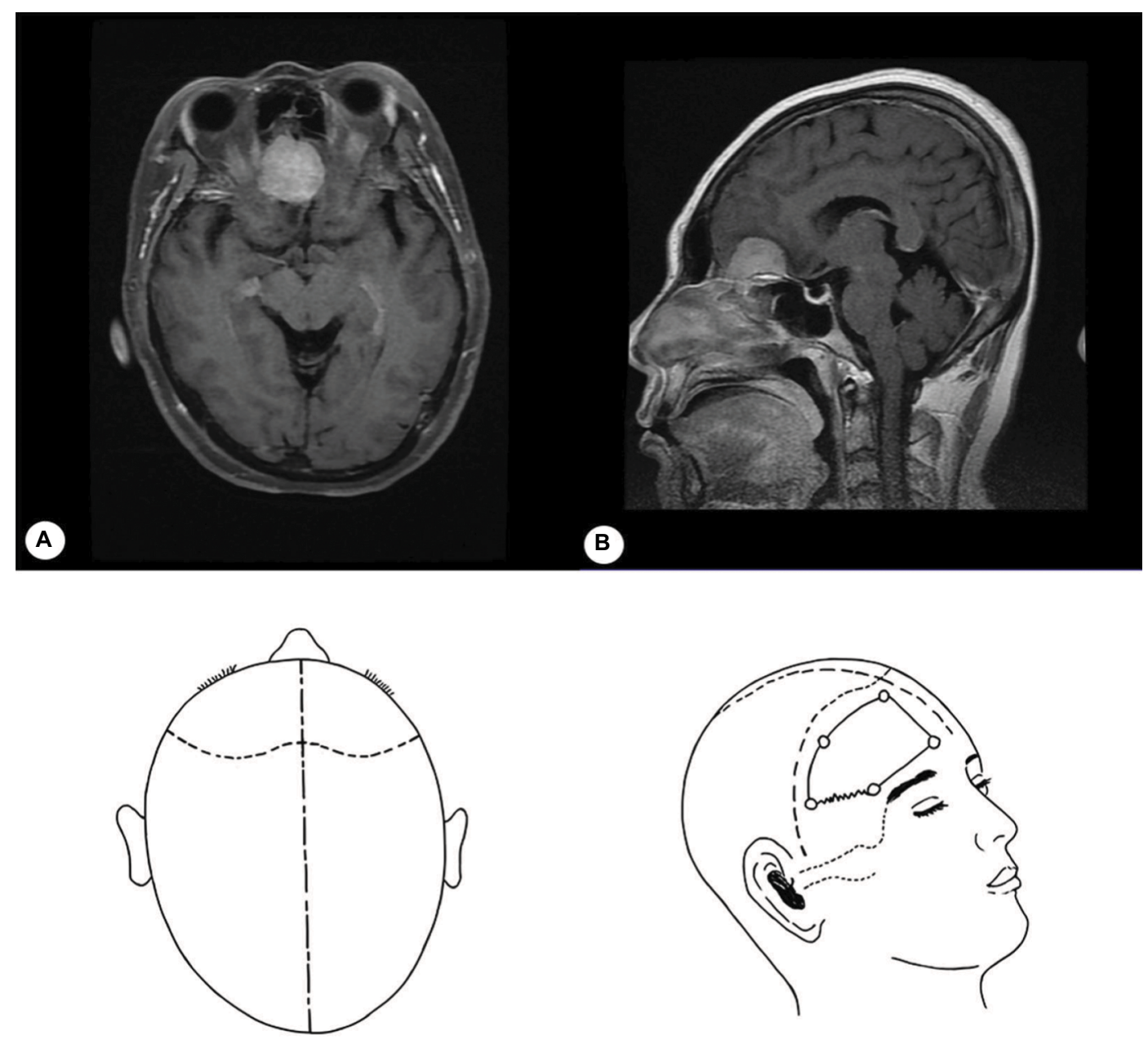

C

Figure I Preoperative cranial magnetic resonance image and design of the surgical incision.

Notes: Spherical occupational lesions in the right anterior cranial fossa, $3 \times 3 \mathrm{~cm}$, with consistent edge patterns (A, B). The surgical opening was an intra-hairline coronal incision, which became a flap (C). The flap was turned forward and downward and fixed with skin retractors to fully expose the skull. The bone window reached the anterior cranial fossa; the right frontal skull was drilled before a bone flap was generated along the bone window margin using a cutter (D). 
The patient laid in a supine position and a Pain Management Generator (PMG-230; Baylis Medical Inc., Montreal, Canada) was attached to the skin of her back through the negative electrode pad. A puncture point, set at $1 / 3$ of the ipsilateral supraorbital margin, was subject to routine disinfection and local anesthesia. A sterile transparent sheath was used to cover the 6-13 MHz linear transducer (probe) of a SonoSite M-Turbo portable ultrasound. Next, aseptic ultrasound gel was placed on and above the patient's eyebrow before the probe was positioned above and parallel to the eyebrow. Cross scanning was performed by gently moving the probe toward the eyebrow, whereby the right supraorbital notch was revealed on the screen (Figure 2A). A $5 \mathrm{~cm}, 21$-gage radiofrequency trocar (PMF-21-50-2; Baylis Medical Inc.) was guided by ultrasound to puncture the supraorbital notch (Figure 2B). The needle core was removed, and there was no bleeding after the piston was withdrawn. A radiofrequency electrode (PMK-21-50; Baylis Medical Inc.) was then introduced. The sensory threshold was determined through $50 \mathrm{~Hz}$ electrical stimulation such that the depth and direction of the puncture needle was fine-tuned until abnormal sensation could be induced by a voltage of $0.1 \mathrm{~V}$. Afterward, the pulsed radiofrequency mode of the pain management generator was initiated to treat the patient with the following specifications: $42^{\circ} \mathrm{C}, 2 \mathrm{~Hz}$ pulsed radiofrequency, 120 seconds in duration, two consecutive treatments.

Just after the pulsed radiofrequency treatment, the VAS decreased from preoperative 9 to postoperative 3 . Then VAS of the patients gradually decreased, and on the fifth day after

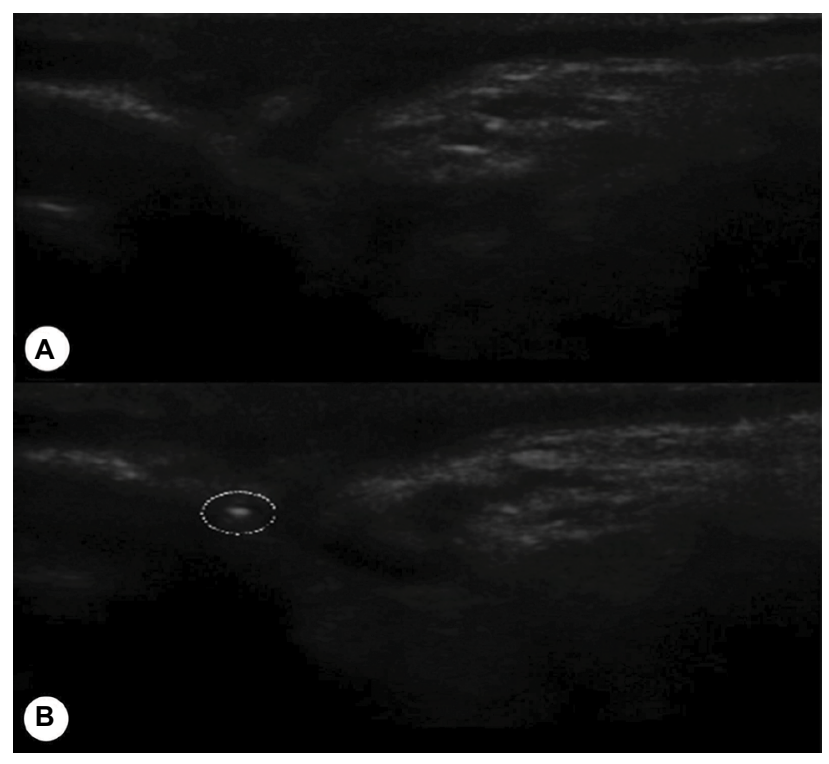

Figure 2 Ultrasound-guided puncture of the supraorbital notch.

Notes: Right supraorbital notch (A). Puncturing the right supraorbital notch with a radiofrequency treatment trocar $(\mathbf{B})$. the treatment, VAS $=0$ was obtained. The subject displayed no pain (VAS $=0$ ) during 12 months of follow-up visits. We evaluated the supraorbital sensory function by testing for light touch, pinprick. Before the treatment, 6 hours, 1 day, and 5 days after the treatment, the responses of the patient were normal in the supraorbital innervated area.

\section{Discussion}

This patient had the following symptoms: 1) pain in the right superior orbital innervated area; 2) right supraorbital notch tenderness; and 3) effective diagnostic block of the right supraorbital nerve. Hence, this case matched the diagnostic criteria for supraorbital neuralgia outlined in the second version of the International Classification of Headache Disorders. ${ }^{10}$ Because the prior therapeutics of medication and conserved nerve block treatment were ineffective, we pursued alternative options. Before more invasive treatments were attempted, we performed nondestructive pulsed radiofrequency and found that this technique produced prominent and lasting efficacy. There are two main differences between this study and previous reports. ${ }^{8,9}$ First, the patient in this study exhibited encouraging efficacy after just one treatment of pulsed radiofrequency; second, instead of using fluoroscopic guidance, we employed ultrasound guidance, which not only explicitly revealed the supraorbital notch but also lacked the side effects of X-rays. Without repeated operations, just one treatment of pulsed radiofrequency allowed the patient long-lasting pain relief. In the meanwhile, we found that this patient had a relatively shorter history compared to patients in other reports. ${ }^{8,9}$ However, whether the length of history is an influencing factor of efficacy of pulsed radiofrequency can only be determined by deeper investigations. Also, future studies with larger sample sizes are necessary to validate whether ultrasound-guided pulsed radiofrequency can be a therapeutic option for supraorbital neuralgia.

Most causes of supraorbital neuralgia are idiomatic or trauma-related. ${ }^{1,2,11}$ In this study, the patient began to experience supraorbital neuralgia on day 3 after a right forehead craniotomy. In 1991, it was reported that some patients developed supraorbital neuralgia after surgery. ${ }^{12}$ Some forehead surgical procedures, such as flap and postoperative pressure dressings, may damage the supraorbital nerve, which could cause supraorbital neuralgia. ${ }^{13}$ It is important to promptly and correctly identify the cause of this disease, which is crucial for the provision of targeted therapy as early as possible. During craniotomies, neurosurgeons should employ strategies to protect the supraorbital nerve and prevent supraorbital neuralgia. ${ }^{14,15}$ 
The patient in this study exhibited no postoperative side effects of nerve damage, demonstrating that it is safe to employ pulsed radiofrequency to treat supraorbital neuralgia. Vallejo et al proposed that pulsed radiofrequency treatment influences the expression level of many pain-related genes to effectively relieve hyperalgesia, and we previously reported that pulsed radiofrequency reduced neuropathic pain behavior through upregulation of glia cell line-derived neurotrophic factor expression. ${ }^{16,17}$ We speculate that the possible pain control mechanism of pulsed radiofrequency might be the selective inhibition of the pain transduction pathways, and of course, the actual mechanism of pulsed radiofrequency treatment is not clear yet. ${ }^{17}$ This is different from traditional radiofrequency ablation, which causes destructive damage to the nerve in a temperature-dependent manner to block the transduction of pain and facilitate pain relief. ${ }^{18}$ Pulsed radiofrequency is a minimally invasive technique that requires no open surgery or implants, is simple to perform, and can be accomplished in an outpatient setting. ${ }^{5-7}$ As such, pulsed radiofrequency treatment represents a future trend for pain treatment.

Consistent with the treatment principles for other pain diseases, we adopted a policy of stepwise ascension in the level of destructiveness when treating this patient with supraorbital neuralgia. Specifically, medication was first attempted, and if it was found ineffective, medication combined with a nerve block treatment was then used. If medication combined with a nerve block treatment was ineffective, nondestructive pulsed radiofrequency was used. If this technique was ineffective, more invasive options would be employed. Fortunately, pulsed radiofrequency generated satisfactory efficacy for the patient and spared her from more invasive procedures such as nerve stimulation, neurolysis, and nerve ablation. The policy of stepwise ascension provided prominent and lasting pain relief at a relatively small cost.

Our results revealed that pulsed radiofrequency is a potentially promising treatment for supraorbital neuralgia. However, it is necessary to perform a quantitative assessment and comparative studies of its efficacy, which require prospective randomized controlled studies.

\section{Conclusion}

Pulsed radiofrequency provided prominent and lasting pain relief and produced no apparent complications in a postcraniotomy patient with supraorbital neuralgia who had previously undergone ineffective treatments of medication and nerve block. Our results suggest that pulsed radiofrequency is a nondestructive and minimally invasive treatment involving simple procedures and may be a promising therapeutic technique for the treatment of supraorbital neuralgia. Studies of this treatment deserve the attention of the research community.

\section{Acknowledgments}

The authors sincerely thank all those who contributed to this work but did not meet the criteria of authorship. This work was supported by Beijing Municipal Administration of Hospitals Clinical Medicine Development of Special Funding Support (grant no. XMLX201707).

\section{Author contributions}

Conception and design: JN, LF. Acquisition of data: XX, RH. Analysis and interpretation of data: XX, RH, LF. Drafting the manuscript and revising it critically: XX, RH, JN, LF. All authors contributed toward data analysis, drafting and revising the paper and agree to be accountable for all aspects of the work.

\section{Disclosure}

The authors report no conflicts of interest in this work.

\section{References}

1. Sjaastad O, Petersen HC, Bakketeig LS. Supraorbital neuralgia. Vågå study of headache epidemiology. Cephalalgia. 2005;25(4):296-304.

2. Caminero AB, Pareja JA. Supraorbital neuralgia: A clinical study. Cephalalgia. 2001;21(3):216-223.

3. Asensio-Samper JM, Villanueva VL, Pérez AV, et al. Peripheral neurostimulation in supraorbital neuralgia refractory to conventional therapy. Pain Pract. 2008;8(2):120-124.

4. Amin S, Buvanendran A, Park KS, Kroin JS, Moric M. Peripheral nerve stimulator for the treatment of supraorbital neuralgia: A retrospective case series. Cephalalgia. 2008;28(4):355-359.

5. Fang L, Jingjing L, Ying S, Lan M, Tao W, Nan J. Computerized tomography-guided sphenopalatine ganglion pulsed radiofrequency treatment in 16 patients with refractory cluster headaches: Twelve- to 30-month follow-up evaluations. Cephalalgia. 2016;36(2):106-112.

6. Luo F, Wang T, Shen Y, Meng L, Lu J, Ji N. High voltage pulsed radiofrequency for the treatment of refractory neuralgia of the infraorbital nerve: A prospective double-blinded randomized controlled study. Pain Physician. 2017;20(4):271-279.

7. Shi Y, Wu W. Treatment of neuropathic pain using pulsed radiofrequency: A meta-analysis. Pain Physician. 2016;19(7):429-444.

8. Brooks AK, Duncan-Azadi CR. Treatment of posttraumatic supraorbital neuralgia using pulsed radiofrequency ablation. A A Case Rep. 2014;3(12):169-170.

9. Dua A, Shepherd J, Bahadur S, Martin D. (472) Supraorbital neuralgia treatment with radio frequency ablation. J Pain. 2016;17(4):S92.

10. Headache Classification Subcommittee of the International Headache Society. The International Classification of Headache Disorders: 2nd edition. Cephalalgia. 2004;24(Suppl 1):9-160.

11. Sjaastad O, Stolt-Nielsen A, Pareja JA, Fredriksen TA, Vincent M. Supraorbital neuralgia. On the clinical manifestations and a possible therapeutic approach. Headache. 1999;39(3):204-212.

12. Klein DS, Schmidt RE. Chronic headache resulting from postoperative supraorbital neuralgia. Anesth Analg. 1991;73(4):490-491.

13. Vacas S, van de Wiele B. Designing a pain management protocol for craniotomy: A narrative review and consideration of promising practices. Surg Neurol Int. 2017;8:291. 
14. Shimizu S, Osawa S, Utsuki S, Oka H, Fujii K. Course of the bony canal associated with high-positioned supraorbital foramina: An anatomic study to facilitate safe mobilization of the supraorbital nerve. Minim Invasive Neurosurg. 2008;51(2):119-123.

15. Mathias RN, Lieber S, de Aguiar PH, Maldaun MV, Gardner P, Fernandez-Miranda JC. Interfascial dissection for protection of the nerve branches to the frontalis muscles during supraorbital trans-eyebrow approach: An anatomical study and technical note. J Neurol Surg B Skull Base. 2016;77(3):265-270.
16. Jia Z, Ren H, Li Q, Ji N, Luo F. Pulsed radiofrequency reduced neuropathic pain behavior in rats associated with upregulation of GDNF expression. Pain Physician. 2016;19(2):49-58.

17. Vallejo R, Tilley DM, Williams J, Labak S, Aliaga L, Benyamin RM. Pulsed radiofrequency modulates pain regulatory gene expression along the nociceptive pathway. Pain Physician. 2013;16(5):E601-E613.

18. Mikeladze G, Espinal R, Finnegan R, Routon J, Martin D. Pulsed radiofrequency application in treatment of chronic zygapophyseal joint pain. Spine J. 2003;3(5):360-362.

\section{Publish your work in this journal}

The Journal of Pain Research is an international, peer reviewed, open access, online journal that welcomes laboratory and clinical findings in the fields of pain research and the prevention and management of pain. Original research, reviews, symposium reports, hypothesis formation and commentaries are all considered for publication.

\section{Dovepress}

The manuscript management system is completely online and includes a very quick and fair peer-review system, which is all easy to use. Visit http://www.dovepress.com/testimonials.php to read real quotes from published authors. 